INPLASY

PROTOCOL

To cite: Liu et al. The Safety and Efficacy of Astragalus for treating diabetic foot ulcers. A protocol for systematic review and meta-analysis. Inplasy protocol 2020110059. doi: 10.37766/inplasy2020.11.0059

Received: 14 November 2020

Published: 15 November 2020

Corresponding author: Yi Zhou

6524118@qq.com

Author Affiliation:

Chengdu university of TCM

Support: NSFC.

Review Stage at time of this submission: Preliminary searches.

Conflicts of interest:

The authors have no conflict of interest to disclose.

\section{The Safety and Efficacy of Astragalus for treating diabetic foot ulcers. A protocol for systematic review and meta-analysis}

\author{
Liu, DH¹; Zhong, YM²; Yan, H³; Hu, YZ4; Chen, YZ5; Zhou, Y6.
}

Review question / Objective: The aim of this meta-analysis of randomized controlled trials is to evaluate the efficacy and safety of effectiveness and safety of Astragalus for treating diabetic foot ulcers during the past 10 years.

Condition being studied: Diabetic foot ulcers are the most common complication in diabetic patients. The annual incidence of foot ulceration among people with diabetes is about 9.26 million. About $80 \%$ of nontraumatic lowerextremity amputations are presented with foot ulceration, which provides a portal for infection. Furthermore, diabetic patients who suffer from foot ulcers encounter financial burden on health five times higher than those without foot ulcers. Astragalus as a Chinese herbal medicine has been reported in many publications that it can treat diabetic foot ulcers by increasing the number of body fibroblasts, promote cell proliferation, reducing the activity of galactosidase in ulcers, improving cell aging and delaying cell aging. However, there is currently no systematic review of the safety and effectiveness of Astragalus in the treatment of diabetic foot ulcer. Therefore, we propose a protocol for a systematic review to evaluate the effectiveness and safety of in the treatment of diabetic foot ulcers.

INPLASY registration number: This protocol was registered with the International Platform of Registered Systematic Review and Meta-Analysis Protocols (INPLASY) on 15 November 2020 and was last updated on 15 November 2020 (registration number INPLASY2020110059).

\section{INTRODUCTION}

Review question / Objective: The aim of this meta-analysis of randomized controlled trials is to evaluate the efficacy and safety of effectiveness and safety of
Astragalus for treating diabetic foot ulcers during the past 10 years.

Condition being studied: Diabetic foot ulcers are the most common complication in diabetic patients. The annual incidence 
of foot ulceration among people with diabetes is about 9.26 million. About $80 \%$ of nontraumatic lower-extremity amputations are presented with foot ulceration, which provides a portal for infection. Furthermore, diabetic patients who suffer from foot ulcers encounter financial burden on health five times higher than those without foot ulcers. Astragalus as a Chinese herbal medicine has been reported in many publications that it can treat diabetic foot ulcers by increasing the number of body fibroblasts, promote cell proliferation, reducing the activity of galactosidase in ulcers, improving cell aging and delaying cell aging. However, there is currently no systematic review of the safety and effectiveness of Astragalus in the treatment of diabetic foot ulcer. Therefore, we propose a protocol for a systematic review to evaluate the effectiveness and safety of in the treatment of diabetic foot ulcers.

\section{METHODS}

Participant or population: The studies will involve adult participants who meet the diagnostic criteria for diabetic foot ulcer. All eligible study participants will be included in this review, regardless of gender, race or occupation. Study participants who are pregnant, breastfeeding, menstruating or suffering from other serious illnesses will be excluded.

Intervention: We will include all randomized controlled trials which uses Astragalus alone(e.g. oral administration, topical use) or in combination with other medical interventions.

Comparator: The control group was treated with commonly used western medicine (such as Buflomedil, Urokinase, Ozagrel, Recombinant Human Growth Hormone,DLThioctic acid etc.), placebo or no intervention measures.

Study designs to be included: Only randomized controlled trials (RCTs) can be included. Observation studies, animal experiment, case report, review, and metaanalysis are excluded.

Eligibility criteria: Participants/population The studies will involve adult participants who meet the diagnostic criteria for diabetic foot ulcer. All eligible study participants will be included in this review, regardless of gender, race or occupation. Study participants who are pregnant, breastfeeding, menstruating or suffering from other serious illnesses will be excluded. Interventions /exposure we will include all randomized controlled trials which uses Astragalus alone(e.g. oral administration, topical use) or in combination with other medical interventions. Comparators/Control: The control group was treated with commonly used western medicine (such as Buflomedil, Urokinase, Ozagrel, Recombinant Human Growth Hormone, DL-Thioctic acid etc.), placebo or no intervention measures. Types of study to be included Only randomized controlled trials (RCTs) can be included. Observation studies, animal experiment, case report, review, and meta-analysis are excluded.

Information sources: The following databases will be searched from 1st January 2010 to September 2020: The Cochrane Library, Pubmed, EMBASE, Web of Science, China National Knowledge Infrastructure and Wanfang Data. All the English and Chinese publications be searched without any restriction of countries.

Main outcome(s): The rate of healing based on the Wagner classification.

Quality assessment / Risk of bias analysis: The methodological quality of RCTs will be assessed by Cochrane risk of bias. It includes generation of the allocation sequence; concealment of the allocation sequence; blinding; attrition and exclusions; other generic sources of bias; biases specific to the trial design (such as crossover or cluster randomized trials); and biases that might be specific to a clinical specialty. Any disagreement will be addressed by discussion among authors. 
Strategy of data synthesis: The analysis and synthesis of data will be conducted by RevMan 5.4.1. The random-effects model and the fixed-effects model will be applied, when 12 is more than $50 \%$ and 12 is less than $50 \%$ respectively. Risk ratio with $95 \%$ confidence interval (Cl). will be used to determine dichotomous data and weighted mean differences (with $95 \%$ Cl) or standardized mean differences $(95 \%) \mathrm{Cl}$ will be used to analyze the continuous data.

Subgroup analysis: Subgroup analysis will be implemented according to administration types, and different outcome measures when there is substantial heterogeneity.

Sensibility analysis: If necessary, a sensitivity analysis will be performed.

Country(ies) involved: China.

Keywords: diabetic foot ulcer, Astragalus, meta-analysis.

Contributions of each author:

Author 1 - Donghao Liu Conceptualization; Methodology; Project administration; Writing - original draft; Writing - review \& editing.

Email: 306734620@qq.com

Author 2 - Yanmei Zhong Conceptualization; Methodology; Project administration; Writing - original draft; Writing - review \& editing.

Email: 1422311457@qq.com

Author 3 - Hang Yan - Suggest amendments.

Email: 675929486@qq.com

Author 4 - Yuanzhang Hu - Software.

Email: 1334756497@qq.com

Author 5 - Yuzhu Chen - Data collection, sorting and reporting.

Email: 1121014514@qq.com

Author 6 - Yi Zhou - Conceptualization; Funding acquisition; Project administration; Validation.

Email: 6524118@qq.com 